\title{
Cholinergic Modulation of an Acetylcholine Receptor-Like Antigen on the Surface of Chick Ciliary Ganglion Neurons in Cell Culture
}

\author{
Martin A. Smith, Joseph F. Margiotta, Alfredo Franco, Jr., Jon M. Lindstrom, ${ }^{1}$ and Darwin K. Berg \\ Department of Biology, University of California at San Diego, La Jolla, California 92093
}

\begin{abstract}
Chick ciliary ganglion neurons have a membrane component that shares an antigenic determinant with the "main immunogenic region" of the alpha subunits in nicotinic $\mathrm{ACh}$ receptors from skeletal muscle and electric organ. Ultrastructural studies on antibody binding in the ganglion have shown that the crossreacting antigen on the neuron surface is located predominantly in synaptic membrane. Biochemical studies have shown that the cross-reacting component has a number of other properties expected for the ganglionic nicotonic $\mathrm{ACh}$ receptor and that it is distinct from the $\alpha$-bungarotoxin binding component in the tissue. Here we show that ciliary ganglion neurons grown in dissociated cell culture express a similar component that crossreacts with monoclonal antibodies to $\mathrm{ACh}$ receptors, and that the number of antibody-binding sites on the neurons can be modulated by exposure to cholinergic agonists and a protein neurotoxin that reversibly inhibits $\mathrm{ACh}$ receptors on the neurons. In most, though not all, cases, levels of $\mathrm{ACh}$ sensitivity associated with the neurons are specifically comodulated in parallel with the changes in number of antibody binding sites. The results suggest that at least a portion of the cross-reacting sites on the surface of ciliary ganglion neurons is likely to represent nicotinic $\mathrm{ACh}$ receptors. The fact that in some instances levels of $\mathrm{ACh}$ sensitivity can be altered without changing the number of cross-reacting sites, however, leaves open the possibility that not all of the sites are associated with receptors or that the neurons can alter the proportion of receptors that is functional.
\end{abstract}

In contrast to the nicotinic acetylcholine receptor (AChR) of muscle and electric organ, little is known about the structure and regulation of nicotinic $\mathrm{AChRs}$ on neurons. Lack of suitable probes has proved to be a major obstacle in this case. $\alpha$-Bungarotoxin ( $\alpha$-Bgt), which binds tightly and specifically to muscle $\mathrm{AChR}$, also recognizes a membrane component on many neurons having nicotinic $A C h R s$, but the relationship of the toxin binding component to the receptor on neurons is unclear. In several cases, the evidence suggests that the toxin-binding component is distinct from the functional AChR on neurons. Thus, chick ciliary ganglion neurons have nicotinic AChRs and highaffinity $\alpha$-Bgt binding sites, but $\alpha$-Bgt fails to block ACh function on the neurons (Ravdin and Berg, 1979), and the toxin-binding sites appear to be largely excluded from postsynaptic membrane on the neurons (Jacob and Berg, 1983; Loring et al., 1985). In addition, the levels of ACh sensitivity and numbers of toxin

Received June 20, 1985; revised Aug. 23, 1985; accepted Aug. 26, 1985.

We thank Dr. Susan Hochschwender for purifying mAb 35, and Dannielle Pellegrin and John Cooper for expert technical assistance. Grant support was provided by the National Institutes of Health (NS 12601 and NS 11323). The Muscular Dystrophy Association, the Alexander Onassis Charitable Foundation, the Los Angeles and California Chapters of the Myasthenia Gravis Foundation, and the American Heart Association with funds contributed in part by the California Heart Association. M.A.S. is a Muscular Dystrophy postdoctoral fellow;

J.F.M. is a National Research Service postdoctoral fellow.

Correspondence should be addressed to Darwin K. Berg.

${ }^{1}$ Present address: The Salk Institute, P.O. Box 85800, San Diego, CA 92138

Copyright (c) 1986 Society for Neuroscience 0270-6474/86/040946-08\$02.00/0 binding sites can be independently regulated on the neurons in cell culture (Smith et al., 1983), indicating that the toxin-binding site is unlikely to represent the functional, synaptic AChR on the neurons. A similar conclusion has been reached for the pheochromocytoma cell line PC12, for which immunological studies (Patrick and Stallcup, 1977) and developmental studies (Mitsuka and Hatanaka, 1983) suggest that the toxin-binding component is distinct from the functional nicotinic AChR on the cells.

Recently, we have used anti-AChR monoclonal antibodies (mAbs) to identify a component in chick ciliary ganglia that shares an antigenic determinant with the "main immunogenic region" (MIR) of AChR alpha subunits in muscle and electric organ. The MIR-like component has several properties expected for a nicotinic AChR on the neurons. It is an integral membrane component that sediments in detergent extracts primarily as a species of $10 \mathrm{~S}$ (Smith et al., 1985), a value close to that observed for AChR monomers from skeletal muscle and electric organ (Changeux et al., 1984). It binds to concanavalin A, which is known to block AChR function on the neurons (Messing et al., 1984). The component is present in detergent extracts of ciliary ganglia and sympathetic ganglia (tissues known to contain nicotinic AChRs), but not in extracts of heart, liver, spinal cord, retina, or dorsal root ganglia (Smith et al., 1985). The greatest increase in specific activity of the component in the ciliary ganglion occurs between embryonic days 8 and 12 , a time when synapses are being consolidated on the neurons (Landmesser and Pilar, 1972,1978). Ultrastructural studies in ciliary ganglia show that the $\mathrm{mAb}$ binding sites present on the surface of the neurons are located predominantly in synaptic membrane (Jacob et al., 1984). The MIR-like component is distinct from the $\alpha$-Bgt-binding component in the ganglion, since toxin sites have a different surface distribution (Jacob and Berg, 1983), and toxin binding sites and $\mathrm{mAb}$ binding sites can be precipitated separately from ganglion extracts (Smith et al., 1985).

Though the MIR-like component is a strong candidate for the nicotinic AChR on ciliary ganglion neurons, a definitive demonstration has been lacking because cross-reacting mAbs do not block AChR function either in muscle (Lindstrom et al., 1983) or in ciliary ganglia (Smith et al., 1985). To examine further the relationship between the MIR-like component and the nicotinic AChR in this case, we tested the ability of cholinergic agents to comodulate the levels of anti-MIR mAb surface binding and $\mathrm{ACh}$ sensitivity of the neurons. Some of these results have been described in a preliminary account (Berg et al., 1985).

\section{Materials and Methods Cell cultures}

Ciliary ganglia from $8 \mathrm{~d}$ chick embryos were dissociated and grown in cell culture at $37^{\circ} \mathrm{C}$ on a substratum of collagen and lysed fibroblasts as previously described (Nishi and Berg, 1981a). Unless otherwise indicated, cultures contained about $10^{4}$ neurons either in $16 \mathrm{~mm}$ wells for $\mathrm{mAb}$ binding experiments or in $35 \mathrm{~mm}$ dishes for electrophysiological or autoradiographic studies. Culture medium consisted of Eagle's min- 


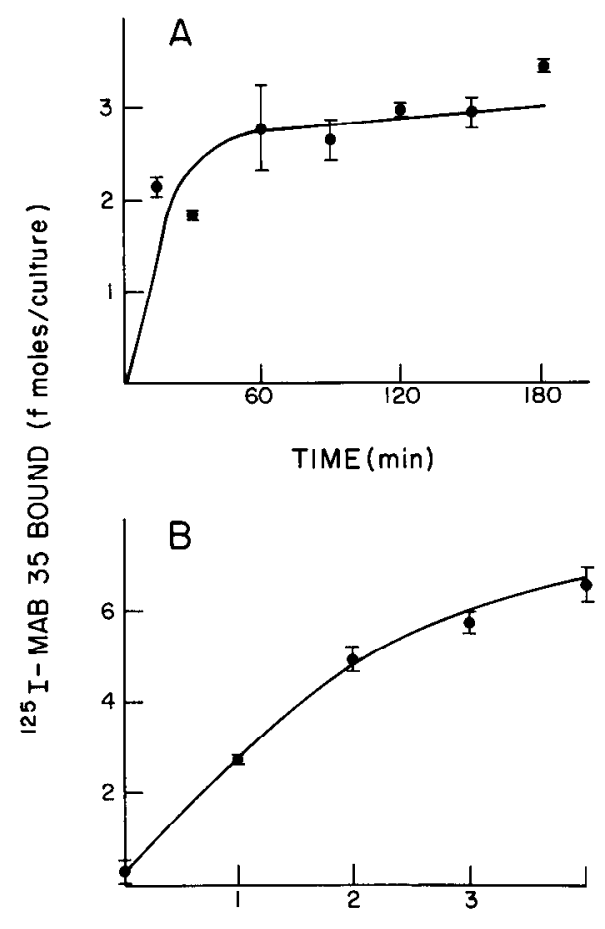

DISSOCIATED GANGLIA PER CULTURE

Figure 1. Time dependence and proportionality to number of neurons for $\mathrm{mAb} 35$ binding. Specific binding of ${ }^{125} \mathrm{I}-\mathrm{mAb} 35$ was determined for cultures of ciliary ganglion ncurons under standard conditions, varying either the time of labeling as indicated $(A)$ or the number of dissociated ciliary ganglia (ca. $6 \times 10^{3}$ neurons/ciliary ganglion) used to prepare the cultures $(B)$. Each determination represents the mean $\pm \mathrm{SE}$ for three cultures. Similar results were obtained in two other experiments.

imal essential medium (MEM) containing 10\% (vol/vol) heat-inactivated horse serum, 50 units $/ \mathrm{ml}$ penicillin, and $50 \mu \mathrm{g} / \mathrm{ml}$ streptomycin. Media supplements included either embryonic chick eye extract (3\% $\mathrm{vol} / \mathrm{vol}$; "eye medium"), $\mathrm{KCl}\left(25 \mathrm{~mm}\right.$ final concentration; " $\mathrm{K}^{+}$medium"), or both ("K+/eye medium"), as previously described (Nishi and Berg, 1981b). Unless otherwise indicated, cultures received eye medium, and the medium was replaced at 2 to $3 \mathrm{~d}$ intervals.

For chronic exposure of cultures to cholinergic ligands, eye medium was prepared with the indicated drugs, sterile filtered, and either supplied to the cells on days 2, 4, and 6 after plating, or as indicated in the figure legends. For acute exposure, cultures were incubated with the drug in eye medium for $1 \mathrm{hr}$ at $37^{\circ} \mathrm{C}$ on day 7 . Unless otherwise indicated, antibody-binding studies were carried out on 7-d-old cultures, and electrophysiological studies on 6- or 7-d-old cultures.

\section{Monoclonal antibodies}

Hybridoma cell lines were obtained by fusing myeloma cell lines with spleen cells from rats immunized with purified muscle or electric organ AChR protein. Anti-MIR mAbs 6, 35, and 203 were raised against receptors from Torpedo (Tzartos and Lindstrom, 1980) and Electrophorus electric organ (Tzartos et al., 1981) and human skeletal muscle (Tzartos et al., 1983), respectively. mAb 112 was raised against AChR from Torpedo electric organ and is of the same antibody subclass as $\mathrm{mAb} 35$ but of different specificity: It recognizes the beta and gamma subunits, but not the alpha subunit, of the receptor (Gullick and Lindstrom, 1983). Stocks were prepared by ammonium sulfate precipitation of hybridoma culture media. mAb 35 was purified further by chromatography on DEAE Affigel blue (S. Hochschwender, unpublished observations), and was radioiodinated by a modified chloramine $T$ method as previously described for $\alpha$-Bgt (Lindstrom et al., 1981) to produce ${ }^{125} \mathrm{I}-\mathrm{mAb} 35$ with initial specific activities of $2-3 \times 10^{18} \mathrm{cpm} /$ mol. ${ }^{125} \mathrm{I}-\mathrm{mAb} 35$ was stored at $4^{\circ} \mathrm{C}$ in PBS containing $0.1 \%$ sodium azide and $10 \mathrm{mg} / \mathrm{ml} \mathrm{BSA}$.

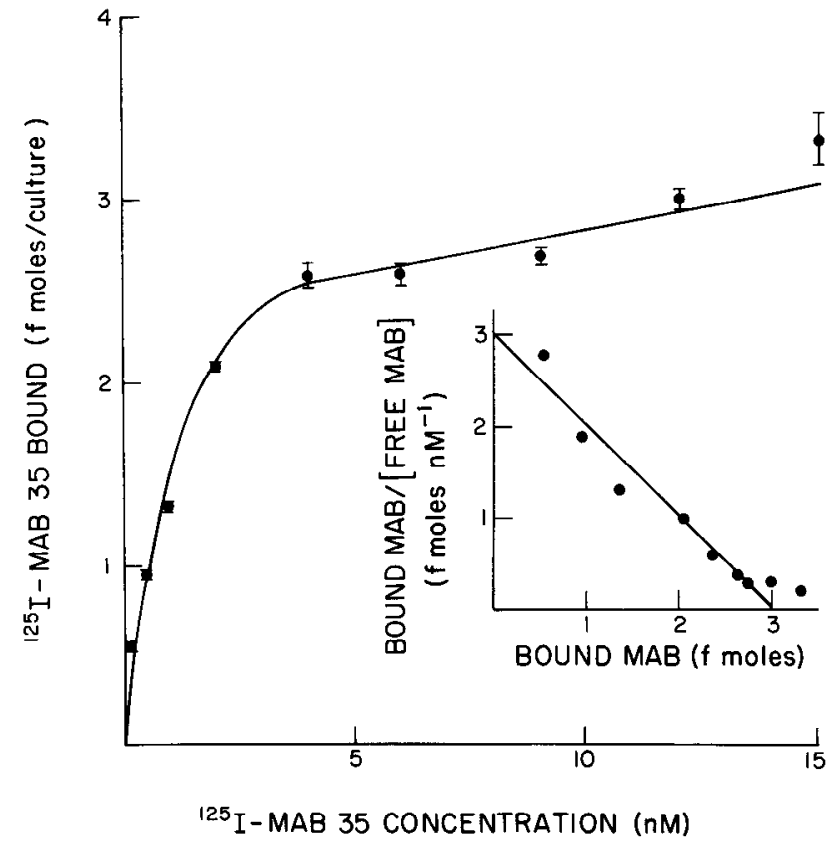

Figure 2. Concentration dependence of mAb 35 binding. Cultures of ciliary ganglion neurons were incubated with the indicated concentrations of ${ }^{125} \mathrm{I}-\mathrm{mAb} 35$ under standard labeling conditions and analyzed for bound $\mathrm{mAb}$. Each point represents the mean $\pm \mathrm{SE}$ of three cultures. Scatchard analysis of the data (inset) indicated a $\mathrm{K}_{\mathrm{d}}$ of $0.97 \mathrm{nM}$ in this experiment. Similar results were obtained in three other experiments.

\section{$m 4 b 35$ binding}

Cultures were rinsed once at room temperature with rinse solution containing $2 \mathrm{mg} / \mathrm{ml} \mathrm{BSA} \mathrm{(1} \mathrm{ml} /$ well; Smith et al., 1983) and then incubated for $1 \mathrm{hr}$ at room temperature in $0.20 \mathrm{ml}$ of solution with the same composition containing $5 \mathrm{nM}{ }^{125} \mathrm{I}-\mathrm{mAb} 35$ (unless otherwise indicated). The labeled cultures were then rinsed $4 \times$ and scraped in 0.5 ml of $0.6 \mathrm{~N} \mathrm{NaOH}$ for quantitating ${ }^{125}$ I with a gamma counter. Nonspecific binding, determined by including a 30 - to 50 -fold excess of unlabeled $\mathrm{mAb} 35$ in the incubation with ${ }^{25}$ I-mAb 35, usually constituted $20-30 \%$ of total binding and was subtracted to yield specific binding, unless otherwise indicated.

\section{Other procedures}

Neurotransmitter sensitivities were measured by using intracellular recording techniques to determine the change in membrane conductance induced by either ACh or GABA pressure-applied to the neurons from a nearby pipette. The assay was set up in the laboratory by $J$. Margiotta and has been described in detail elsewhere (Margiotta and Berg, 1986; McEachern et al., 1985; Smith et al., 1983).

Autoradiographic studies of $\mathrm{mAb} 35$ binding to the neurons in culture were carried out by growing the neurons on glass coverslips as previously described (Jacob et al., 1984) with the indicated drugs. Cultures were labeled with ${ }^{125} \mathrm{I}-\mathrm{mAb} 35$ and rinsed as described above, fixed in $2 \%$ formaldehyde and $2 \%$ glutaraldehyde for $1 \mathrm{hr}$ at room temperature, rinsed and mounted on glass slides, and processed for autoradiography (Smith et al., 1983). Lactic acid dehydrogenase assays were performed as described by Nishi and Berg (1981a).

\section{Materials}

Culture media components were obtained from Grand Island Biological Co., and tissue extracts and culture media were prepared as previously described (Nishi and Berg, 1981a). Bungarus multicinctus venom was purchased from Miami Serpentarium, and $\alpha$-Bgt and Bgt 3.1 were purified from the venom as previously described (Ravdin and Berg, 1979; Ravdin et al., 1981). $d$-Tubocurarine chloride, carbamylcholine chloride (carbachol), acetylcholine chloride, neostigmine bromide, and nicotine sulfate were purchased from Sigma. Trimethaphan camsylate Ro 2-2222 was the generous gift of Hoffmann-La Roche. 


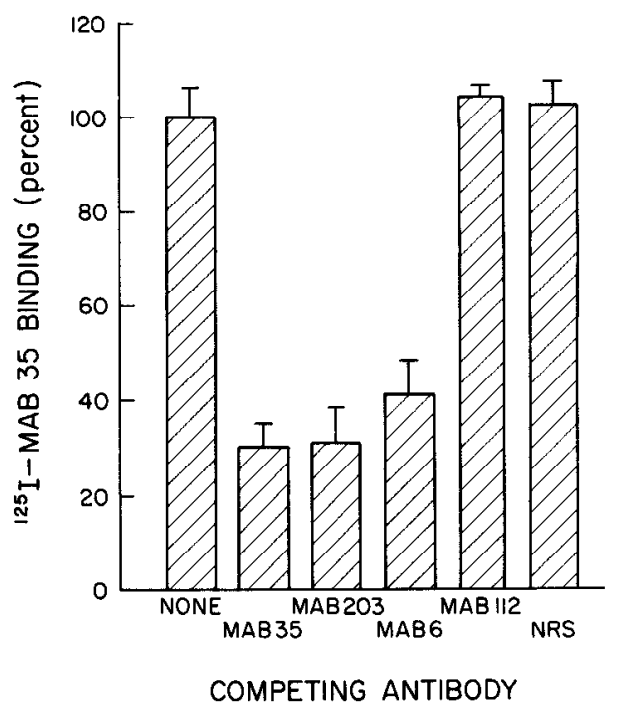

Figure 3. Specificity of antibody binding. The indicated antibodies were incubated at $0.50 \mu \mathrm{M}$ along with ${ }^{125} \mathrm{I}-\mathrm{mAb} 35$ in the standard labeling assay, and the cultures were analyzed for bound ${ }^{125} \mathrm{I}-\mathrm{mAb} 35$. Each point represents the mean $\pm S E$ of results from four experiments (three cultures per experiment), except for $\mathrm{mAb} 112$, where the mean represents two experiments. Nonspecific binding has not been subtracted.

\section{Results}

\section{mAb 35 binding}

The amount of cross-reacting MIR-like component on the surface of ciliary ganglion neurons in culture was determined by incubating cultures with ${ }^{125} \mathrm{I}-\mathrm{mAb} 35$, rinsing the cultures, and measuring the amount of bound radioactivity. At room temperature, maximum binding was achieved within $60 \mathrm{~min}$, and the amount of binding was proportional to the number of neurons present (Fig. 1). Near-saturation of antigen occurred with $5 \mathrm{nM}{ }^{125} \mathrm{I}-\mathrm{mAb} 35$ (Fig. 2). Scatchard analysis of the data from four separate experiments revealed a class of high-affinity $\mathrm{mAb}$ 35 binding sites with an apparent equilibrium dissociation constant $\left(K_{d}\right)$ of $1.49 \pm 0.47 \mathrm{~nm}{ }^{125} \mathrm{I}-\mathrm{mAb} 35$ binding in the cultures was both saturable and specific, since other anti-MIR mAbs,

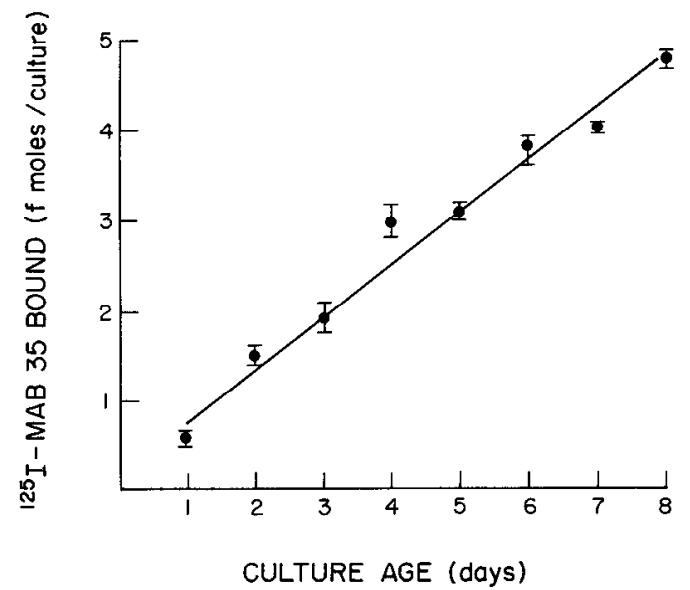

Figure 4. Increase in the number of MIR-like sites with time in culture. At the indicated times, cultures of ciliary ganglion neurons were assayed for specific ${ }^{125} \mathrm{I}-\mathrm{mAb} .35$ binding. Each determination represents the mean $\pm \mathrm{SE}$ for nine cultures combined from three experiments, except for days 1,2 , and 8 , which represent six cultures from two experiments in each case.

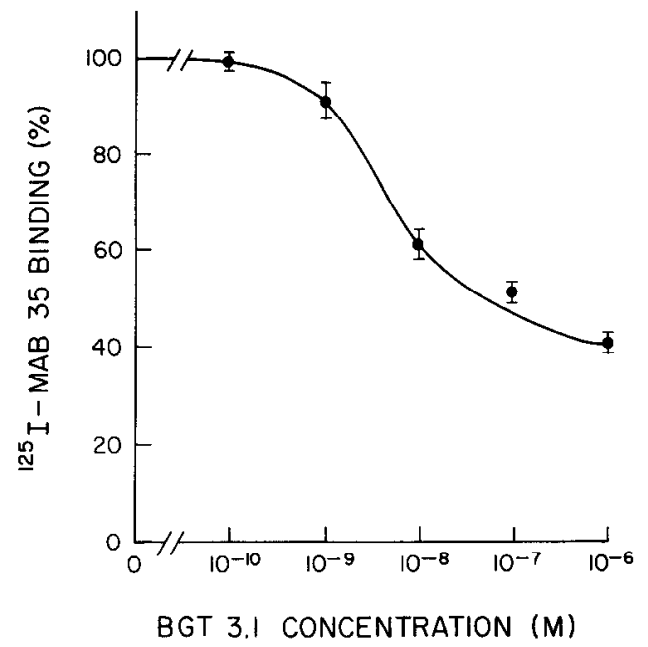

Figure 5. Concentration dependence for acute Bgt 3.1 modulation of MIR-like sites. Cultures were incubated with the indicated concentration of Bgt 3.1 for $1 \mathrm{hr}$ at $37^{\circ} \mathrm{C}$, rinsed, and assayed for specific ${ }^{125} \mathrm{I}-$ $\mathrm{mAb} 35$ binding. The results are expressed as the percentage of binding levels obtained with sister cultures not exposed to Bgt 3.1. Each determination represents the mean \pm SE for six cultures combined from two experiments, except for the value for $10^{-8} \mathrm{M}$ Bgt 3.1, which represents the mean of 30 determinations compiled from 10 cxpcriments.

such as $\mathrm{mAb} 6$ and $\mathrm{mAb} 203$, blocked the binding to the same extent as did unlabeled mAb 35, while no blockade of ${ }^{125} \mathrm{I}-\mathrm{mAb}$ 35 binding occurred with mAb 112 (an mAb of the same subclass as $\mathrm{mAb} 35$ but with different specificity) or with nonimmune rat serum at a comparable concentration of immunoglobulin (Fig. 3). The number of MIR-like sites on the neuronal surface increased linearly with culture age over the $8 \mathrm{~d}$ period examined (Fig. 4).

\section{Acute modulation}

Bgt 3.1 is a protein neurotoxin that reversibly blocks the $\mathrm{AChR}$ function of ciliary ganglion neurons (Ravdin and Berg, 1979). The toxin is present as a minor component in the venom of $B$.

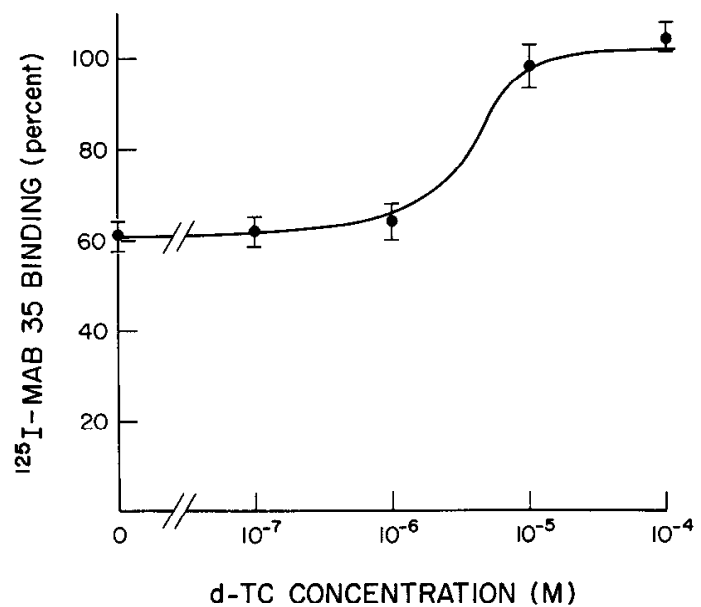

Figure 6. Concentration dependence for $d$-tubocurarine protection against the acute Bgt 3.1 effect. Cultures were incubated with the indicated concentrations of $d$-tubocurarine along with $10^{-8} \mathrm{M} \mathrm{Bgt} 3.1$ for $1 \mathrm{hr}$ at $37^{\circ} \mathrm{C}$, rinsed, and assayed for specific ${ }^{125} \mathrm{I}-\mathrm{mAb} 35$ binding. The results are expressed as the percentage of the binding obtained for sister cultures not exposed to either Bgt 3.1 or $d$-tubocurarine. Each determination represents the mean $\pm \mathrm{SE}$ of six cultures combined from two experiments, except for $10^{-4} \mathrm{M}$ dTC, which represents 12 cultures from four experiments. 


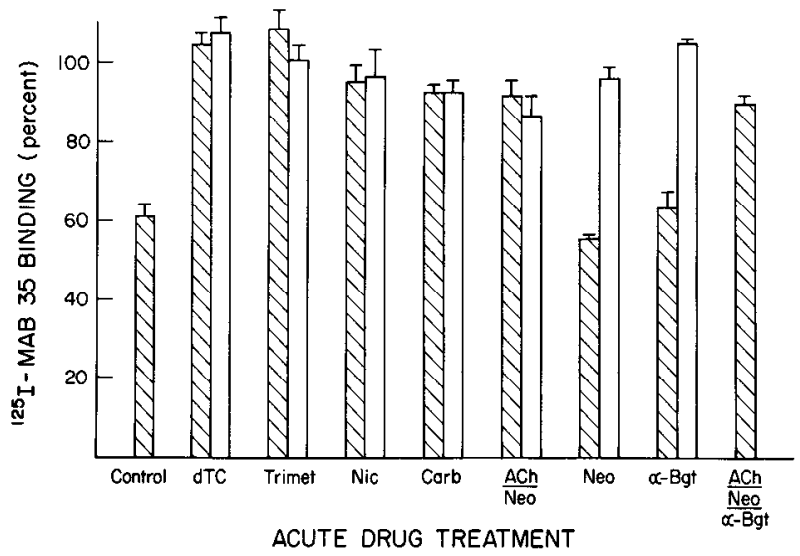

Figure 7. Effects of cholinergic ligands on the acute modulation by Bgt 3.1. Cultures were incubated with (hatched hars) or without (open hars) $10^{-8} \mathrm{M}$ Bgt 3.1 and the indicated ligands, rinsed, and assayed for specific ${ }^{125} \mathrm{I}-\mathrm{mAb} 35$ binding. The results are expressed as the percentage of binding obtained with sister cultures not exposed to Bgt 3.1 or cholinergic ligands. Each determination represents the mean \pm SE of either nine cultures from three experiments (hatched bars) or six cultures from two experiments (open bars) except for Control ( 30 cultures, 10 experiments) and dTC alone (12 cultures, four experiments). Control, No cholinergic ligand added to the Bgt $3.1 ; d T C, 10^{-4} \mathrm{M} d$-tubocurarine; Trimet, $10^{-4} \mathrm{M}$ trimethaphan; Nic, $10^{-4} \mathrm{M}$ nicotine; Carb, $10^{-3} \mathrm{M}$ carbachol; $A C h / N e o, 10^{-3} \mathrm{M}$ ACh $+10^{-5} \mathrm{M}$ neostigmine; Neo, $10^{-5} \mathrm{M}$ neostigmine; $\alpha$-Bgt, $10^{-6} \mathrm{M} \alpha$-Bgt; $A C h / N e o / \alpha-B g t, 10^{-3} \mathrm{M} \mathrm{ACh}+10^{-5}$ $\mathrm{M}$ neostigmine $+10^{-6} \mathrm{M} \alpha$-Bgt.

multicinctus and can be separated from the more common $\alpha$-Bgt in the same venom (Ravdin and Berg, 1979; Ravdin et al., 1981). Exposure of ciliary ganglion neurons to Bgt 3.1 for $1 \mathrm{hr}$ at $37^{\circ} \mathrm{C}$ causes the loss of about half of the MIR-like sites available to bind ${ }^{125} \mathrm{I}-\mathrm{mAb} 35$ subsequently (Fig. 5). This reduction in number of MIR-like sites does not represent competition by the toxin for the antigen: Co-incubation of Bgt 3.1 with ${ }^{125} \mathrm{I}-\mathrm{mAb}$ 35, either while labeling the solubilized MIR-like component in ganglion extracts or while labeling cultures at $4^{\circ} \mathrm{C}$, where

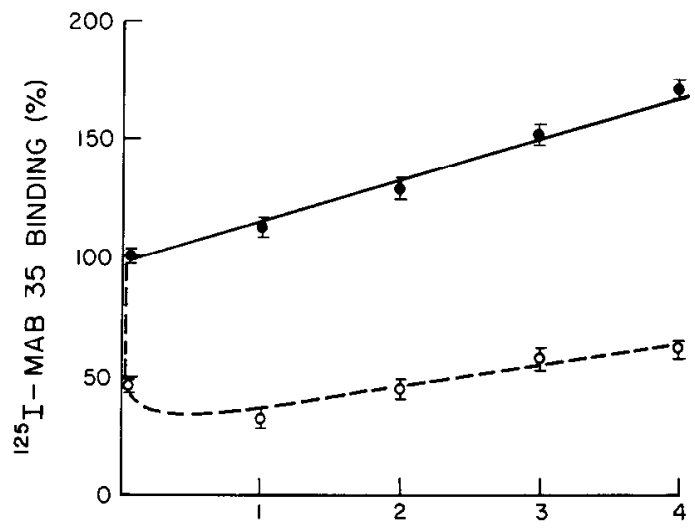

BGT 3.I EXPOSURE (days)

Figure 8. Chronic modulation of MIR-like sites by Bgt 3.1. Cultures grown in normal medium (eye medium) for $3 \mathrm{~d}$ were transferred to medium containing $10^{-8} \mathrm{M}$ Bgt 3.1 and were assayed for specific ${ }^{225} \mathrm{I}-$ $\mathrm{mAb} 35$ binding at the indicated times (open circles, dashed line). Sister cultures were maintained in normal medium and assayed in parallel (filled circles, solid line). Results are expressed as the percentage of the binding obtained for cultures at $3 \mathrm{~d}+1 \mathrm{hr}$ in medium lacking Bgt 3.1. Each determination represents the mean \pm SE for 12 cultures taken from four experiments, except for days 2 and 3 , which represent nine cultures from three experiments in each case.

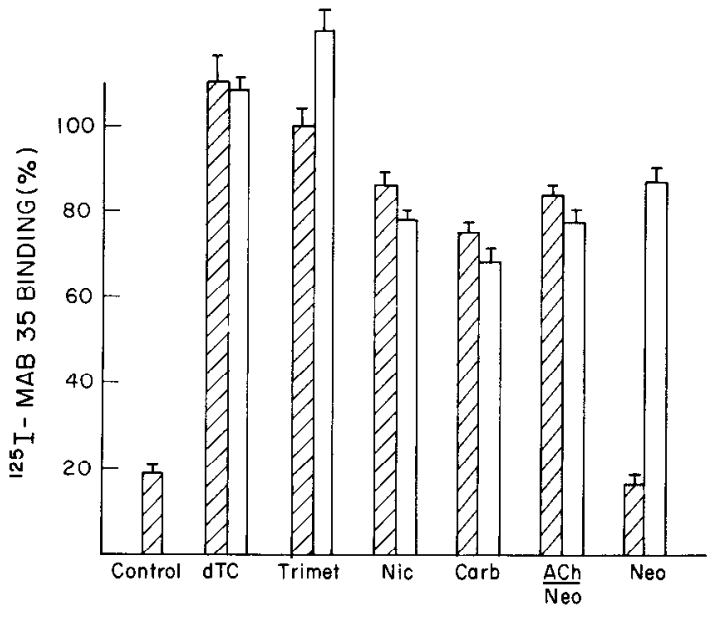

CHRONIC DRUG TREATMENT

Figure 9. Fffects of cholinergic ligands on the chronic modulation by Bgt 3.1. Neurons were grown with (hatched bars) or without (open bars) $10^{-8} \mathrm{M}$ Bgt 3.1 and the indicated ligands from days 2-7 in culture, then rinsed and assayed for specific ${ }^{125} \mathrm{I}-\mathrm{mAb} 35$ binding. Results are expressed as the percentage of the binding obtained with sister cultures not exposed either to Bgt 3.1 or cholinergic ligands. Each determination represents the mean $\pm \mathrm{SE}$ for nine cultures combined from three experiments, except for Control ( 15 cultures, five experiments), Carb alone and $\mathrm{ACh} / \mathrm{Neo}$ alone (11 cultures, four experiments each), Neo with Bgt 3.1 (six cultures, two experiments), and Neo alone (12 cultures, four experiments). The drug concentrations are the same as those given in Figure 7.

modulation of surface sites cannot occur, does not reduce the amount of specific antibody binding observed (Smith et al., 1985, and unpublished observations). Increasing the concentration of ${ }^{125} \mathrm{I}-\mathrm{mAb} 35$ threefold in the standard assay fails to detect additional sites after acute exposure of the neurons to Bgt 3.1 (data not shown).

Bgt 3.1 inhibits AChR function on the neurons (Ravdin and Berg, 1979) at toxin concentrations similar to those effective here in modulating the MIR-like component. In addition, Bgt 3.1 blocks $\alpha$-Bgt binding to the neurons (Ravdin et al., 1981). Indirect evidence (see Discussion) indicates that these two effects of Bgt 3.1 arc achicved by interaction of the toxin with two distinct classes of binding sites, one being the $\alpha$-Bgt binding site and the other possibly being the AChR (Ravdin et al., 1981). We were interested in determining whether modulation of the MIR-like component by Bgt 3.1 depends on interaction with a cholinergic site and, if so, which class of sites might be important. Accordingly, we tested the ability of various cholinergic ligands to protect the MIR-like component from Bgt 3.1-induced modulation. Co-incubation of the cultures with $10^{-5} \mathrm{M}$ $d$-tubocurarine during treatment with $10^{-8} \mathrm{M} \mathrm{Bgt} 3.1$ completely blocked the modulation (Fig. 6). Other cholinergic ligands werc also effective. Trimethaphan and nicotine at $10^{-4} \mathrm{M}$, and carbachol and $\mathrm{ACh}$ at $1 \mathrm{~mm}$, were each able to protect against the Bgt 3.1 effect (Fig. 7). The protection by ACh cannot be ascribed to the accompanying neostigmine (present to prevent hydrolysis of the ACh by esterases in the culture medium) because the same concentration of neostigmine alone had no effect against the Bgt 3.1-induced modulation. In contrast to the cholinergic ligands, $\alpha$-Bgt at $10^{-6} \mathrm{M}$ provided no protection against the Bgt 3.1 effect and did not interfere with the protection conferred by ACh. Since the concentration of $\alpha$-Bgt used represented a 50 fold excess over that needed to saturate the $\alpha$-Bgt binding site (Ravdin et al., 1981), the results indicate that the cholinergic site at which Bgt 3.1 modulates the MIR-like component is different from the $\alpha$-Bgt binding site on the neurons. None of 


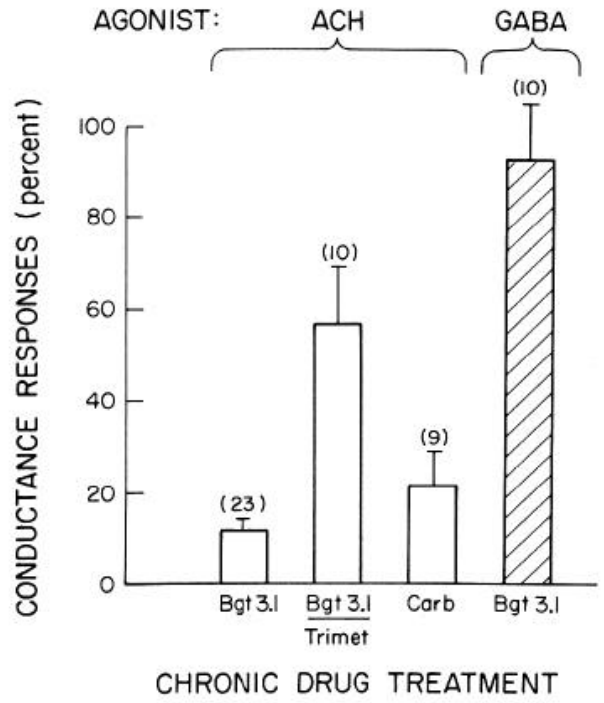

Figure 10. Modulation of $\mathrm{ACh}$ sensitivity. Cultures were grown either in medium containing $10^{-8} \mathrm{M}$ Bgt 3.1 (Bgt 3.1) or $10^{-8} \mathrm{M}$ Bgt 3.1 and $10^{-4} \mathrm{M}$ trimethaphan (Bgt 3.1/Trimet), or $10^{-3} \mathrm{M}$ carbachol (Carb), as described in Figure 9. After rinsing extensively (eight changes of medium over a $1 \mathrm{hr}$ period), the neurons were tested for ACh sensitivity (open bars) or GABA sensitivity (hatched bar), as indicated. The rinsing procedure was adequate to restore the ACh sensitivity almost completely after blockade by $10^{-8} \mathrm{M}$ Bgt 3.1 for $1 \mathrm{hr}$ at $37^{\circ} \mathrm{C}$ and all of the $\mathrm{ACh}$ sensitivity after desensitization by $1 \mathrm{~mm}$ carbachol for $10 \mathrm{~min}$ at $37^{\circ} \mathrm{C}$. Each determination represents the mean \pm SE for the number of neurons indicated in parentheses and and is expressed as the percentage of the value obtained for neurons in sister cultures grown in normal medium (eye medium). In all cases, results were compiled for neurons from at least two separate culture platings. The mean overall $100 \%$ values for ACh and GABA induced conductance changes were $32 \pm 3 \mathrm{nS}(n=$ $8)$ and $30 \pm 5 \mathrm{nS}(n=8)$, respectively.

the ligands had any significant effect by themselves on the number of ${ }^{125} \mathrm{I}-\mathrm{mAb} 35$ binding sites during the $1 \mathrm{hr}$ incubation at $37^{\circ} \mathrm{C}$.

\section{Chronic modulation}

Chronic exposure of the neurons to $10^{-8} \mathrm{M}$ Bgt 3.1 caused an initial rapid decrease in the number of surface sites available for ${ }^{125} \mathrm{I}-\mathrm{mAb} 35$ binding. This was followed by a small and slow increase in the number of sites over a period of days (Fig. 8). Both cholinergic agonists and antagonists were again able to protect against modulation of the MIR-like component by chronic exposure to Bgt 3.1 (Fig. 9). Protection by agonists, however, appeared to be incomplete. The explanation emerged from testing the agonists alone: Chronic exposure to $1 \mathrm{~mm}$ carbachol caused a $30 \%$ loss in the number of ${ }^{125} \mathrm{I}-\mathrm{mAb} 35$ binding sites on the surface of the neurons (Fig. 9). The decrease did not result from a change in cell size, since neurons grown with and without $1 \mathrm{~mm}$ carbachol appeared similar in size and had identical levels of the cytoplasmic marker enzyme, lactic acid dehydrogenase (data not shown). Thus, the number of MIR-like sites remaining after carbachol treatment was the same with or without Bgt 3.1, indicating that the agonist not only provided complete protection against the Bgt 3.1 effect, but also induced modulation of the sites independently. Smaller effects were observed with nicotine and $\mathrm{ACh}$. The ganglionic antagonist trimethaphan appeared to induce a small increase in the total number of MIR-like sites on the cells.

\section{Comodulation of ACh sensitivity}

The levels of ACh sensitivity associated with the neurons after drug treatment were tested to determine whether they changed in parallel with the number of MIR-like surface sites. Chronic

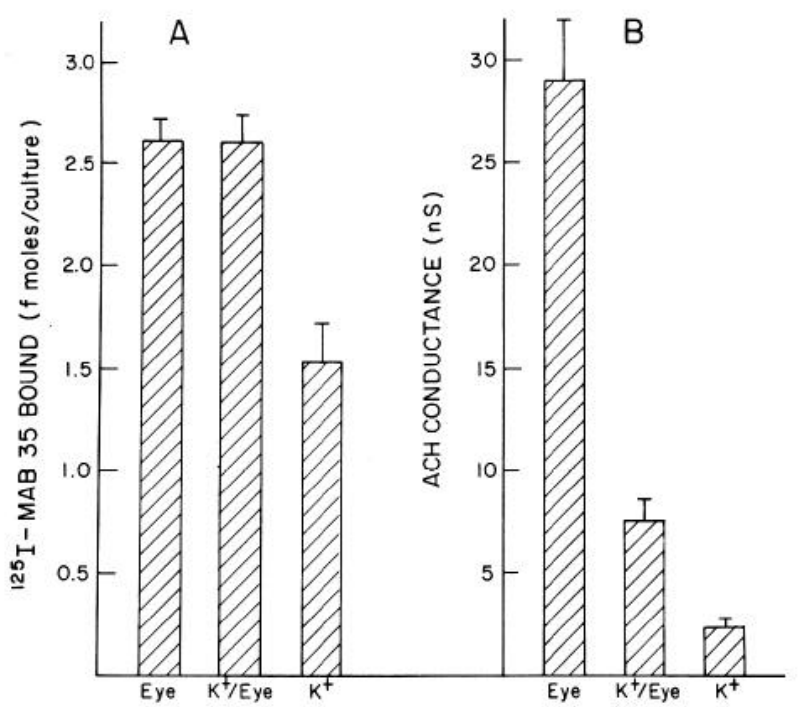

Figure 11. Differential modulation of ACh sensitivity and MIR-like sites by growth conditions. $A$, Neurons were grown either in eye medium (eye), $\mathrm{K}^{+} /$eye medium $\left(K^{+} /\right.$Eye $)$or $\mathrm{K}^{+}$medium $\left(K^{+}\right)$and assayed for specific ${ }^{125} \mathrm{I}-\mathrm{mAb} 35$ binding. $B$, For comparison, the levels of $\mathrm{ACh}$ sensitivity are shown for neurons grown under the same conditions (taken from Smith et al., 1983, Fig. 2A).

exposure to Bgt 3.1 followed by extensive rinsing to remove bound Bgt 3.1 resulted in levels of ACh sensitivity that were reduced to approximately the same extent as the numbers of MIR-like sites (Fig. 10). The reduction in ACh sensitivity is
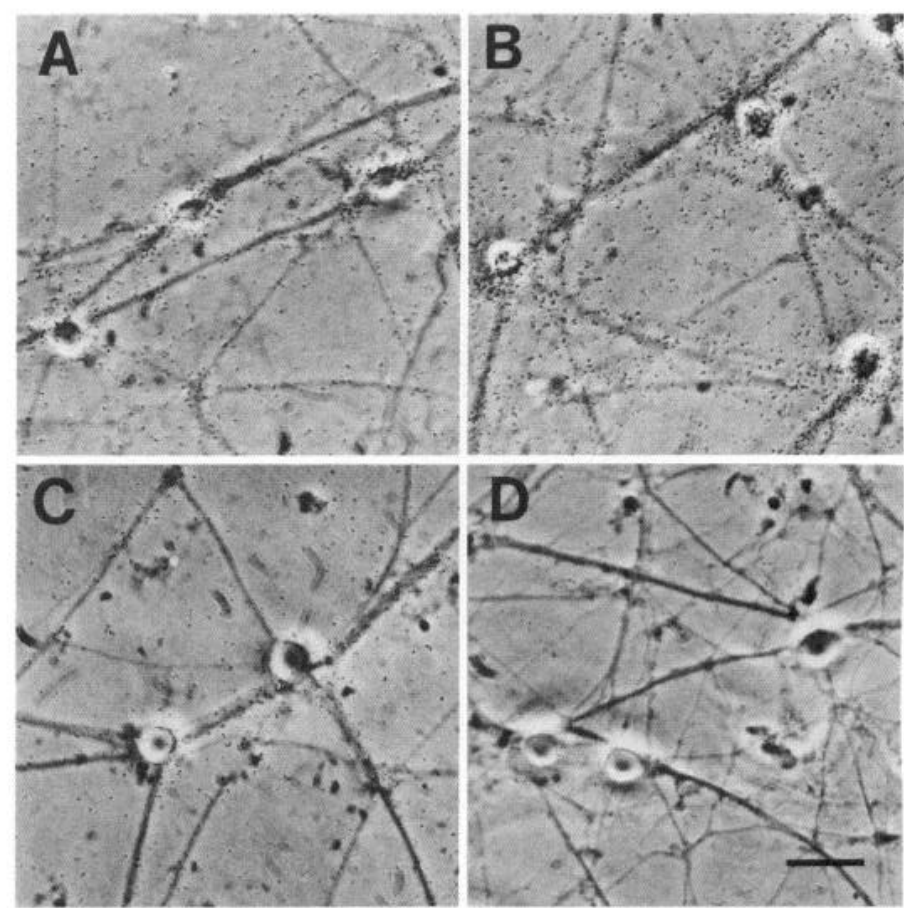

Figure 12. Autoradiographic comparison of mAb 35 binding to neuronal cell bodies grown under different conditions. Cultures were grown for $7 \mathrm{~d}$ in eye medium $(A, D)$ or $\mathrm{K}^{+} /$eye medium $(B)$ or for $2 \mathrm{~d}$ in eye medium followed by $5 \mathrm{~d}$ in medium supplemented with $10^{-8} \mathrm{M}$ Bgt 3.1 (C). The cultures were then rinsed, labeled with ${ }^{125} \mathrm{I}-\mathrm{mAb} 35(A-C)$ or ${ }^{125} \mathrm{I}-\mathrm{mAb} 35$ plus a 50 -fold excess of unlabeled mAb $35(D)$, and processed for autoradiography. Phase-contrast optics were used, and the focal plane was adjusted to reveal grains in the emulsion layer. Calibration bar, $20 \mu \mathrm{m}$. 
likely to represent modulation rather than simple inhibition, since acute exposure of the neurons to $\mathrm{Bgt} 3.1$, followed by a similar pattern of rinsing, restored nearly all of the original $\mathrm{ACh}$ sensitivity (Ravdin and Berg, 1979; J. Margiotta, unpublished observations). The Bgt 3.1 effect was specific in that no reduction was observed in the levels of GABA sensitivity. Co-incubation of the neurons in $10^{-4} \mathrm{M}$ trimethaphan along with the Bgt 3.1 resulted in a partial protection against the modulation of $\mathrm{ACh}$ sensitivity. Chronic exposure to $1 \mathrm{~mm}$ carbachol (in the absence of Bgt 3.1), followed by extensive rinsing to remove bound carbachol and to allow for recovery from desensitization, resulted in a nearly fourfold reduction in levels of $\mathrm{ACh}$ sensitivity (Fig. 10). Acute exposure to $1 \mathrm{~mm}$ carbachol (10 $\mathrm{min}$ ), followed by the same pattern of rinsing, produced no significant decrease in ACh sensitivity compared to that found for control neurons, indicating that desensitization had been reversed by the protocol (data not shown). The qualitative agreement in specific comodulation of levels of ACh sensitivity and numbers of MIR-like sites is consistent with the MIR-like component representing the nicotinic AChR on the neurons.

\section{Exceptions to comodulation.}

In some instances, changes in the levels of ACh sensitivity associated with the neurons do not correlate with the numbers of ${ }^{125} \mathrm{I}-\mathrm{mAb} 35$ binding sites. Acute exposure of the neurons to $10^{-7}$ M Bgt 3.1 causes a $50 \%$ reduction in the number of $\mathrm{mAb} 35$ binding sites (Fig. 5), and 1-2 hr of rinsing does not increase the number of sites detected (M. A. Smith, unpublished observations). The rinsing does achieve, however, a nearly complete recovery of $\mathrm{ACh}$ sensitivity, as cited above. A more clear-cut discrepancy is detected by examining the effects of different growth conditions. Previous studies have shown that chronic growth in medium supplemented with elevated concentrations of $\mathrm{K}^{+}\left(\mathrm{K}^{+}\right.$/eye medium) leads to reduced levels of ACh sensitivity for the neurons compared to that of neurons grown in normal medium (eye medium), all assayed under standard conditions (Smith et al., 1983). ${ }^{125}$ I-mAb 35 binding is not reduced in parallel under these conditions (Fig. 11). Lack of correlation cannot be accounted for by a selective migration of MIR sites away from the soma when neurons are grown in elevated $\mathrm{K}^{+}$: Autoradiographs of neuronal cultures labeled with ${ }^{125} \mathrm{I}-\mathrm{mAb} 35$ indicate comparable numbers of grains associated with the somata of neurons grown in eye and $\mathrm{K}^{+} /$eye media, though they have nearly a fourfold difference in the levels of soma ACh sensitivity (Fig. 12). The autoradiographs do show a reduced level of soma labeling by ${ }^{125} \mathrm{I}-\mathrm{mAb} 35$ for neurons chronically exposed to $10^{-8} \mathrm{M} \mathrm{Bgt} 3.1$ (Fig. 12), consistent with values for total ${ }^{125} \mathrm{I}-\mathrm{mAb} 35$ binding in the cultures (Figs. 8, 9) and for soma levels of ACh sensitivity (Fig. 10). If neurons from $\mathrm{K}^{+/}$ eye medium are compared with neurons from $\mathrm{K}^{+}$medium (instead of eye medium), a much better correlation is observed between relative levels of ACh sensitivity and numbers of MIRlike sites (Fig. 11).

\section{Discussion}

Previous studies demonstrated that chick ciliary ganglion neurons in dissociated cell culture express a surface component that cross-reacts with mAbs to the MIR of muscle and electric organ AChR alpha subunits (Jacob et al., 1984). The present studies show that the amount of the component on the neurons in culture and the specificity and $K_{\mathrm{d}}$ value for anti-MIR mAb binding are similar to those described for the solubilized MIRlike component in detergent extracts of ciliary ganglia (Smith et al., 1985). Fifteen day embryonic ganglia contain about $5.5 \mathrm{fmol}$ of anti-MIR mAb binding sites, of which $40 \%$, or about $2 \mathrm{fmol}$, would be expected to be on the surface of the cells if surface sites constituted the same fraction in vivo (Jacob et al., 1985) that they do in culture (Smith et al., 1985). Neurons from $8 \mathrm{~d}$ embryonic ganglia maintained in cell culture for $7 \mathrm{~d}$ also have about 2 fmol of surface MIR-like component per ganglion equivalent of neurons (though it should be noted that $15 \mathrm{~d}$ embryonic ciliary ganglia have only half as many neurons as do $8 \mathrm{~d}$ ganglia because of naturally occurring cell death in vivo; Landmesser and Pilar, 1974).

The major finding reported here is that specific modulation of the MIR-like component on ciliary ganglion neurons in culture can be achieved through a cholinergic site. This was shown by the action of Bgt 3.1 on the neurons, by the array of cholinergic ligands that protected against the Bgt 3.1 effect, and by the direct effects of cholinergic agonists alone. In these cases, the levels of ACh sensitivity associated with the neurons were comodulated with the number of MIR-like components on the neuron surface, as expected if the MIR-like component represents the nicotinic AChR on the cells.

Bgt 3.1 has previously been shown to recognize two classes of sites on chick ciliary ganglion neurons. Interaction of Bgt 3.1 with the first class of sites causes the specific and reversible inhibition of nicotinic AChR function on the cells. The inhibition is unaffected by the presence or absence of high concentrations of $\alpha$-Bgt, which does not itself block the neuronal AChRs (Ravdin and Berg, 1979; Ravdin et al., 1981). Interaction of Bgt 3.1 with the second class of sites has the unusual effect of inducing the rapid internalization of $\alpha$-Bgt bound to the cells (Ravdin et al., 1981). The second class of sites is likely to be identical with the $\alpha$-Bgt sites themselves, since saturation of all the sites with $\alpha$-Bgt prevents Bgt 3.1 from inducing internalization of the bound $\alpha$-Bgt, and at low temperatures, when internalization cannot occur, competition can be demonstrated between the binding of ${ }^{125} \mathrm{I}-\alpha$-Bgt and Bgt 3.1 (Ravdin et al., 1981). Recent electrophoretic and amino acid composition data (R. Loring and R. Zigmond, personal communication) indicate that Bgt 3.1 is identical to $k$-bungarotoxin (Dryer and Chiappinelli, 1983) and toxin $F$ (Loring et al., 1984), all derived from $B$. multicinctus venom. Studies with both $k$-bungarotoxin (Chiappinelli, 1983) and toxin F (Loring et al., 1984) have also indicated two classes of Bgt 3.1 binding sites on ciliary ganglion neurons. In these cases, specific binding of the toxin (using a radiolabeled derivative) in the ganglion was found to be only partially blocked by $\alpha$-Bgt. The remaining sites were comparable in number to the MIR-like sites described here, and may represent nicotinic $\mathrm{AChRs}$ on the neurons.

The mechanism by which Bgt 3.1 modulates the number of MIR-like sites on the neurons is unknown. The site of action, however, is likely to involve the cholinergic site on the nicotinic AChR. The fact that cholinergic agonists and antagonists are able to protect the MIR-like component against modulation by Bgt 3.1 clearly indicates that a cholinergic site is involved. The concentrations of Bgt 3.1 needed for AChR blockade and for modulation of the component are similar. The other Bgt 3.1 binding site, i.e., the site that also binds $\alpha$-Bgt, is unlikely to be important for the modulation of MIR-like sites because $\alpha$-Bgt docs not itsclf modulate the MIR-like component, and even at a 100-fold excess over the Bgt 3.1 concentration, $\alpha$-Bgt is unable to protect against $\mathrm{Bgt}$ 3.1-induced modulation of the component. Moreover, $\alpha$-Bgt is unable to interfere with the ability of cholinergic ligands to protect against the Bgt 3.1-induced modulation. The simplest explanation is that the MIR-likc component is the AChR, and that Bgt 3.1 induces modulation of the MIR-like component by binding to the same site that results in receptor blockade by Bgt 3.1; cholinergic ligands protect against the $\mathrm{Bgt} 3.1$ effect either by competing with $\mathrm{Bgt} 3.1$ for binding or by stabilizing the receptor in a resistant conformation.

The inference that the MIR-like component represents the AChR on the neurons is supported by the observation that chronic exposure of the cells to cholinergic agonists induces a decrease of about $30 \%$ in the number of MIR-like sites. The 
reduction is reminiscent of the commonly occurring phenomenon of agonist-induced down-regulation of receptors, and is similar in magnitude to the decrease in AChRs found with chick myotubes ( $32 \%$ in $72 \mathrm{hr}$ ) after chronic exposure to $0.1 \mathrm{~mm}$ carbachol (Gardner and Fambrough, 1979). The concentrations of agonists used in the present study (1 mM) are similar to those needed to achieve maximal $\mathrm{ACh}$ responses from the neurons (Smith et al., 1983). There is precedence for neuronal nicotinic AChRs having lower affinities for agonist than do muscle nicotinic AChRs (Ascher et al., 1979).

If the MIR-like component on the neurons is the functional nicotinic AChR, changes in the number of MIR-like sites should be accompanied by similar changes in the levels of ACh sensitivity associated with the cells. This expectation was met qualitatively in several instances. Chronic exposure to Bgt 3.1 (followed by rinsing to remove the Bgt 3.1) reduced both the number of MIR-like sites and the levels of ACh sensitivity to less than a fifth of control values. GABA sensitivities were unaffected by chronic exposure to Bgt 3.1, indicating that the modulation of ACh sensitivity was specific. Co-incubation of trimethaphan along with Bgt 3.1 protected against the reduction in MIR-like sites and partially protected against the reduction in ACh sensitivity. Chronic exposure to carbachol reduced both the number of MIR-like sites and the amount of ACh sensitivity, though the latter was depressed to a greater extent. Failure to obtain quantitative agreement in some cases is not surprising in view of the inherent difficulties in using the physiological assay to assess relative numbers of functional receptors.

In three instances, however, discrepancies were observed between ACh sensitivities and MIR-like sites, and these merit further discussion. One of these is the finding that the number of MIR-like sites increases linearly with time in culture (Fig. 4), while the level of $\mathrm{ACh}$ sensitivity reaches a plateau value early on (Smith et al., 1983). Another is the observation that acute exposure to Bgt 3.1, followed by extensive rinsing, results in nearly complete recovery of ACh sensitivity, while the number of MIR-like sites remains at $50 \%$. A number of explanations could account for these first two results. The increase in MIRlike sites with time in culture, for example, might reflect receptors appearing on the neurites that would not score in the physiological assay. The acute modulation of MIR-sites by Bgt 3.1 might represent rearrangement of the sites in the membrane (e.g., clustering of receptors) that sterically hinders $\mathrm{mAb}$ binding without impairing receptor function. (Chronic modulation would represent subsequent internalization of the sites, accompanied by loss of receptor function and $\mathrm{mAb}$ binding.) Explanations of this kind, however, cannot account for the third case, i.e., the substantial discrepancy between the relative levels of ACh sensitivity and numbers of MIR-like sitcs for ncurons grown in cyc medium versus $\mathrm{K}^{+} /$eye medium. Autoradiographs confirmed that comparable labeling of neuronal somata by ${ }^{125} \mathrm{I}-\mathrm{mAb} 35$ was obtained for the two types of cultures, even though a nearly fourfold difference was observed in the levels of ACh sensitivity. Preliminary results from whole-cell patch-clamp experiments have confirmed the approximately fourfold difference in AChinduced conductance changes for neurons grown in eye versus $\mathrm{K}+$ /eye medium, and have also shown that the growth conditions do not cause a change in the single-channel parameters of AChRs on the cells (Margiotta et al., 1985). Though other workers did not report reduced $\mathrm{ACh}$ sensitivity for ciliary ganglion neurons grown in high concentrations of $\mathrm{K}^{+}$(Bader et al., 1982), the methods used there would probably not have detected the fourfold difference described here.

Most of the evidence is consistent with the MIR-like component on ciliary ganglion neurons representing the nicotinic $A C h R$. In the ganglion, the component on the surface of the neurons is located predominantly in synaptic membrane (Jacob et al., 1984). Preliminary results indicate that intracellular MIR- like sites appear to be associated with organelles known to be part of the biosynthetic and regulatory pathways for cell-surface membrane proteins (Jacob et al., 1985). Biochemical studies on tissues from the periphery indicate that it is restricted to tissues known to have ganglionic nicotinic AChRs, and that it has many of the properties expected for the nicotinic AChR on neurons (Smith et al., 1985). A polyclonal rat serum raised against a partially purified MIR-like component from chick brain ( $P$. Whiting and J. Lindstrom, unpublished observations) that is similar to the MIR-like component in ciliary ganglia (Lindstrom et al., 1983) specifically blocks $85 \%$ of the ACh sensitivity associated with ciliary ganglion neurons in cell culture when applied at a 1:100 dilution (J. Stollberg and D. Berg, unpublished observations). The present studies demonstrate that the MIRlike site on ciliary ganglion neurons in cell culture can be modulated by cholinergic agents. In addition, changes in the number of MIR-like sites are usually associated with parallel changes in the levels of $\mathrm{ACh}$ sensitivity associated with the neurons. The fact that some discrepancies exist, however, in the correlation between sensitivity and site number leaves open the possibility that the component does not actually represent the nicotinic $\mathrm{AChR}$, and that the considerable evidence suggesting the contrary is simply coincidence.

An alternative explanation is that only a fraction of the MIRlike sites on the neurons represents functional $\mathrm{AChRs}$, and that the remainder represents either inactive receptors or some other protein. If the surface sites do include inactive $\mathrm{AChRs}$, the results comparing $\mathrm{ACh}$ sensitivities for neurons from different growth conditions would imply that the neurons can alter or regulate the proportion of AChRs that is functional. It will be interesting to determine the types of regulation possible for neuronal nicotinic AChRs, and to obtain additional evidence concerning the relationship of the MIR-like component and the AChR on the neurons.

\section{References}

Ascher, P., W. A. Large, and H. P. Rang (1979) Studies on the mechanism of action of acetylcholine antagonists on rat parasympathetic ganglion cells. J. Physiol. (Lond.) 295: 139-170.

Bader, C. R., D. Bertrand, and A. C. Kato (1982) Chick ciliary ganglion in dissociated cell culture. II. Electrophysiological properties. Dev. Biol. 94: 131-141.

Berg, D. K., M. A. Smith, J. F. Margiotta, and J. M. Lindstrom (1985) Cholinergic modulation of an acetylcholine receptor-like antigen on the surface of chick ciliary ganglion neurons in cell culture. Soc. Neurosci. Abstr. 11: 170.

Changeux, J.-P., A. Devillers-Thiery, and P. Chemouilli (1984) Acetylcholine receptor: An allosteric protein. Science 225: 1335-1345.

Chiappinelli, V. A. (1983) $\kappa$-Bungaroloxin: A probe for the neuronal nicotinic receptor in the avian ciliary ganglion. Brain Res. 277:9-21.

Dryer, S. E., and V. A. Chiappinelli (1983) $\kappa$-Bungarotoxin: An intracellular study demonstrating blockade of neuronal nicotinic receptors by a snake neurotoxin. Brain Res. 289: 317-321.

Gardner, J. M., and D. M. Fambrough (1979) Acetylcholine receptor degradation measured by density labeling: Effects of cholinergic ligands and evidence against recycling. Cell 16: 661-674.

Gullick, W. J., and J. M. I indstrom (1983) Mapping the binding of monoclonal antibodies to the acetylcholine receptor from Torpedo californica. Biochemistry 22: 3312-3320.

Jacob, M. H., and D. K. Berg (1983) The ultrastructural localization of $\alpha$-bungarotoxin binding sites in relation to synapses on chick ciliary ganglion neurons. J. Neurosci. 3: 260-271.

Jacob, M. H., D. K. Berg, and J. M. Lindstrom (1984) Shared antigenic determinant between the Electrophorus acetylcholine receptor and synaptic component on chick ciliary ganglion neurons. Proc. Natl. Acad. Sci. USA 81: 3223-3227.

Jacob, M. H., J. M. Lindstrom, and D. K. Berg (1985) Surface and intracellular distribution of an acetylcholine receptor-like antigen in chick ciliary ganglion neurons. Soc. Neurosci. Abstr. 11: 171.

Landmesser, L., and G. Pilar (1972) The onset and development of 
transmission in the chick ciliary ganglion. J. Physiol. (Lond.) 222: 691-713.

Landmesser, L., and G. Pilar (1974) Synaptic transmission and cell death during normal ganglionic development. J. Physiol. (Lond.) 241: 737-749.

Landmesser, L., and G. Pilar (1978) Interactions between neurons and their targets during in vivo synaptogenesis. Fed. Proc. 37: 2016-2022.

Lindstrom, J., B. Einarson, and S. Tzartos (1981) Production and assay of antibodies to acetylcholine receptors. Methods Enzymol. 74: 432-459.

Lindstrom, J., S. Tzartos, W. Gullick, S. Hochschwender, L. Swanson, P. Sargent, M. Jacob, and M. Montal (1983) Use of monoclonal antibodies to study acetycholine receptors from electric organs, muscle, and brain and the autoimmune response to receptor in myasthenia gravis. Cold Spring Harbor Symp. Quant. Biol. 48: 89-99.

Loring, R. H., V. A. Chiappinelli, R. E. Zigmond, and J. B. Cohen (1984) Characterization of a snake venom neurotoxin which blocks nicotinic transmission in the avian ciliary ganglion. Neuroscience 11 : 989-999.

Loring, R. H., L. M. Dahm, and R. E. Zigmond (1985) Localization of $\alpha$-bungarotoxin binding sites in the ciliary ganglion of the embryonic chick: An autoradiographic study at the light and electron microscopic level. Neuroscience 14: 645-660.

Margiotta, J. F., and D. K. Berg (1982) Functional synapses are established between ciliary ganglion neurons in dissociated cell culture. Nature 296: 152-154.

Margiotta, J. F., and D. K. Berg (in press) Enkephalin and substance $P$ modulate synaptic properties of chick ciliary ganglion neurons in cell culture. Neuroscience.

Margiotta, J. F., D. K. Berg, and V. E. Dionne (1985) Regulation of acetylcholine receptors on chick ciliary ganglion neurons in cell culture. Soc. Neurosci. Abstr. 11: 171.

McEachern, A. E., J. F. Margiotta, and D. K. Berg (1985) GABA receptors on chick ciliary ganglion neurons in vivo and in cell culture. J. Neurosci. 5: 2690-2695.

Messing, A., B. Bizzini, and N. K. Gonatas (1984) Concanavalin A inhibits nicotinic acetylcholine receptor function in cultured chick ciliary ganglion neurons. Brain Res. 303: 241-249.
Mitsuka, M., and H. Hatanaka (1983) Selective loss of acetylcholine scnsitivity in a nerve cell line cultured in hormone-supplemented serum-free medium. J. Neurosci. 3: 1785-1790.

Nishi, R., and D. K. Berg (1981a) Two components from eye tissue that differentially stimulate the growth and development of ciliary ganglion neurons in cell culture. J. Neurosci. 1: 505-513.

Nishi, R., and D. K. Berg (1981b) Effects of high $\mathrm{K}^{+}$concentrations on the growth and development of ciliary ganglion neurons in cell culture. Dev. Biol. 87: 301-307.

Patrick, J., and W. B. Stallcup (1977) Immunological distinction between acetylcholine receptor and the $\alpha$-bungarotoxin binding component on sympathetic neurons. Proc. Natl. Acad. Sci. USA 74:46894692.

Ravdin, P. M., and D. K. Berg (1979) Inhibition of neuronal acetylcholine sensitivity by alpha-toxins from Bungarus multicinctus venom. Proc. Natl. Acad. Sci. USA 76: 2072-2076.

Ravdin, P. M., R. M. Nitkin, and D. K. Berg (1981) Internalization of $\alpha$-bungarotoxin on neurons induced by a neurotoxin that blocks neuronal acetylcholine sensitivity. J. Neurosci. 1: 849-861.

Smith, M. A., J. F. Margiotta, and D. K. Berg (1983) Differential regulation of acetylcholine sensitivity and $\alpha$-bungarotoxin-binding sites on ciliary ganglion neurons in cell culture. J. Neurosci. 3: 23952402.

Smith, M. A., J. Stollberg, D. K. Berg, and J. M. Lindstrom (1985) Characterization of a component in chick ciliary ganglia that crossreacts with monoclonal antibodies to muscle and electric organ acetylcholine receptor. J. Neurosci. 5: 2726-2731.

Tzartos, S. J., and J. M. Lindstrom (1980) Monoclonal antibodies used to probe acetylcholine receptor structure: Localization of the main immunogenic region and detection of similarities between subunits. Proc. Natl. Acad. USA 77: 755-759.

Tzartos, S., D. E. Rand, B. L. Einarson, and J. M. Lindstrom (1981) Mapping of surface structures of Electrophorus acetylcholine receptor using monoclonal antibodies. J. Biol. Chem. 256: 8635-8645.

Tzartos, S., L. Langeberg, S. Hochschwender, and J. Lindstrom (1983) Demonstration of a main immunogenic region on acetylcholine receptors from human muscle using monoclonal antibodies to human receptor. FEBS Lett. 158: 116-118. 